\title{
La antropología social en los contextos mexicano, latinoamericano y universal en la vida y obra de Rodolfo Stavenhagen*
}

\author{
Salomón Nahmad Sitton
}

\section{BREVE SEMBLANZA DESDE MI PERSPECTIVA}

$\mathrm{F}$ ue para mí un honor haber participado en el homenaje a Rodolfo Stavenhagen que le rindieron la Universidad Nacional Autónoma de México (UNAM) y varias instituciones ligadas a la investigación antropológica y a la acción de políticas públicas en favor de las clases populares y, en particular, de los pueblos indígenas de México, del Continente Americano y del mundo, por su gran trayectoria como investigador y como antropólogo comprometido con los derechos humanos y en contra de todas las formas de colonialismo internacional y nacional, de todas las formas de explotación humana que se manifiestan en todas las regiones del mundo; por su gran trabajo para eliminar el racismo, el etnocidio, la esclavitud y la exclusión de miles de pueblos y de comunidades indígenas en los proyectos nacionales que se han implementado en el mundo contemporáneo a través de las organizaciones internacionales - como Naciones Unidas (oNU), la Organización de las Naciones Unidas para la Educación, la Ciencia y la Cultura (unEsCo), la Organización Internacional del Trabajo (OIT), la Universidad de Naciones Unidas-, gracias a su papel activo y de gran relevancia, desde su juventud hasta el presente, como un expositor del gran humanismo que acompaña su vida como defensor de los derechos humanos.

\section{Social Anthropology in the Mexican, Latin American and Universal Context in the Life and Work of Rodolfo Stavenhagen}

\author{
Salomón Nahmad Sitton: Centro de Investigaciones y Estudios Superiores en Antropología Social, unidad Pacífico Sur, \\ Oaxaca, Oaxaca, México \\ snahmad@ciesas.edu.mx
}

Desacatos, núm. 43, septiembre-diciembre 2013, pp. 181-186

\footnotetext{
* Una primera versión de este texto corresponde al discurso presentado en el homenaje "Sociología y Antropología en la obra de Rodolfo Stavenhagen" que le rindió el Instituto de Investigaciones Sociales de la Universidad Nacional Autónoma de México, el 30 de abril de 2013.
} 
Rodolfo en los años cincuenta y haber construido una gran amistad con él a lo largo de los años. En esta relación se entrecruzan nuestros afectos familiares y sobre todo el vínculo de carácter académico y de acciones múltiples para pensar en construir en México una sociedad que elimine la injusticia y la discriminación y que erijamos, como es su propuesta, una sociedad multicultural, multiétnica y multilingüística. A través de las instituciones académicas se han cimentado con el pensamiento y el conocimiento real y objetivo la etnografía, la etnología y la antropología social, de la que Rodolfo es uno de los exponentes más preclaros en el mundo contemporáneo y la cual ha engrandecido a partir de sus reflexiones en el contexto de la antropología mexicana. Los presentes en ese homenaje sin duda lo reconocemos como uno de los pilares de este conocimiento científico aprendido en la ENAH en la segunda y la tercera generaciones, quienes nos encontramos en las aulas y en los seminarios específicos como estudiantes, como compañeros, entre los que quiero destacar a los que han sido y fueron sus grandes amigos: Julio de la Fuente, Gonzalo Aguirre Beltrán, Leonel Durán, Guillermo Bonfil, Enrique Valencia, Margarita
Nolasco, Maurilio Muñoz, Gildardo González, Mercedes Olivera, Carlos Navarrete, Íker Larrauri, Lourdes Arizpe, Claudio Esteva Fabregat, Ángel Palerm, Pedro Carrasco, Ignacio Bernal, que acompañados por otros colegas de América Latina, como Darcy Ribeiro, Julio Cotler y Roberto Cardoso de Oliveira, y amigos profesores de la Escuela como Frederick Katz, John Murra, Alejandro Marroquín, Alfonso Villa Rojas, entre otros, y sobre todo los grandes maestros que nos dieron formación antropológica en esas primeras generaciones, como Juan Comas, Mauricio Swadesh, Pablo Martínez del Río, Alfonso Caso, Paul Kirchhoff, Ricardo Pozas, Arturo Monzón, Fernando Cámara Barbachano, Eusebio Dávalos, Felipe Montemayor y otros grandes y distinguidos maestros que consolidaron la llamada "escuela de antropología mexicana" y de los cuales Rodolfo Stavenhagen es uno de los herederos emblemáticos de lo que se reconoce como el movimiento antropológico más destacado de América Latina. Considero que ese homenaje lo incluye como uno de los impulsores de la nueva antropología mexicana en tanto corriente progresista y transformadora de las realidades sociales mexicanas y mundiales.

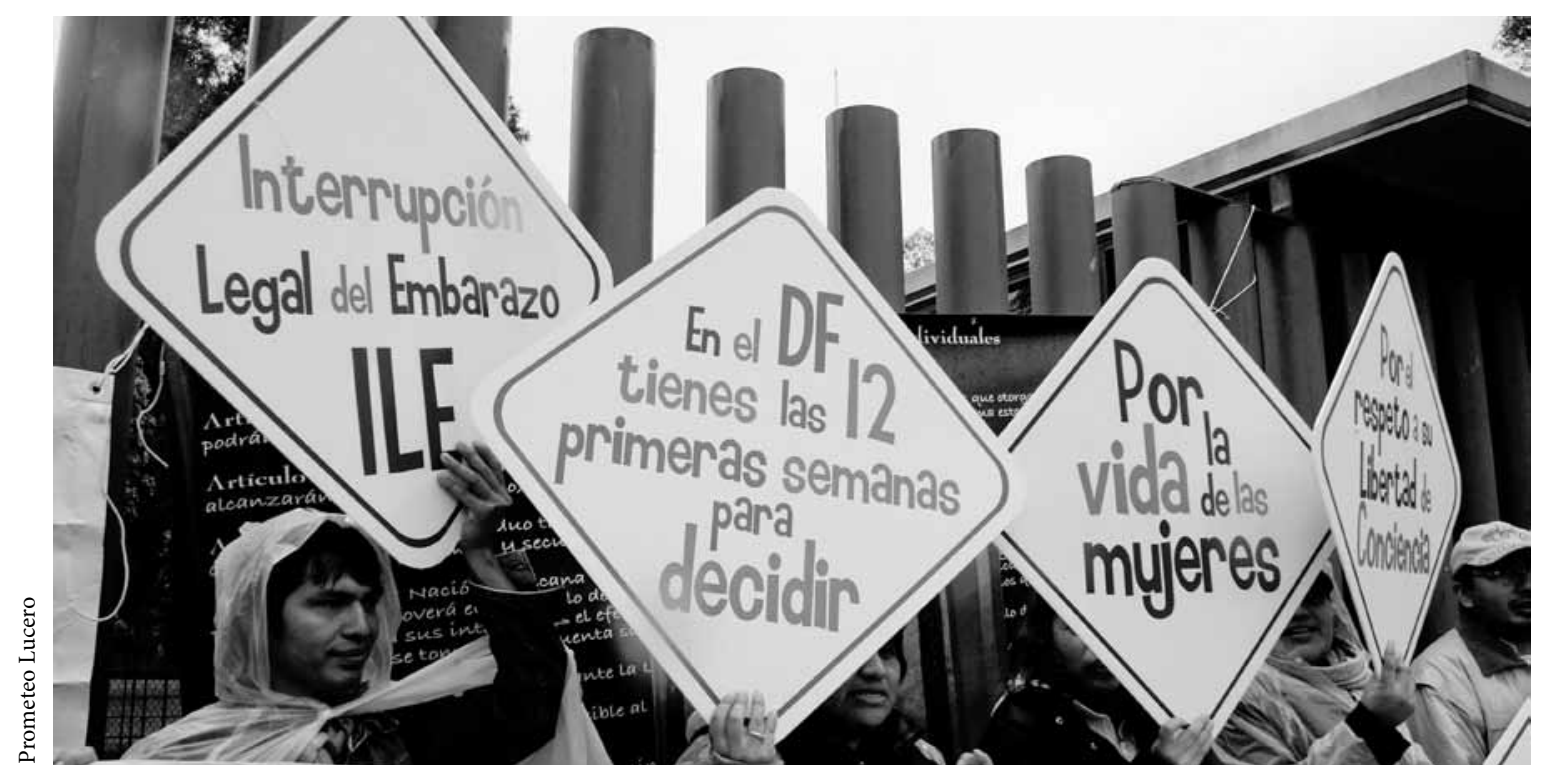

Por el derecho a decidir, San Lázaro, Distrito Federal, 2007. 


\section{RODOLFO COMO UNO DE LOS PILARES DE LA ANTROPOLOGÍA MEXICANA EN LA SEGUNDA MITAD DEL SIGLO XX Y PRINCIPIOS DEL XXI}

No es posible soslayar la posición de Rodolfo Stavenhagen en la antropología crítica de los proyectos de desarrollo, como el de la Comisión del Papaloapan, sobre la estructura de las clases sociales, la continuidad del colonialismo, la aculturación y la integración social, el papel del indigenismo en el siglo $\mathrm{xx}$, sus importantes contribuciones al análisis de la reforma agraria y a las relaciones interétnicas, su gran aportación con el libro Conflictos étnicos y Estado nacional (Stavenhagen, 2000b), en cuyo estudio se incluyeron seis grandes regiones de todos los continentes, en 15 países, que más adelante comentaré. Revisando la enorme contribución científica de Rodolfo al mundo de las ciencias sociales, puedo señalar que su primer trabajo publicado fue el artículo "Aspectos de la antropología social aplicada en la Cuenca del Papaloapan”, en la revista Tlatoani, en 1953, en el que se perfiló de manera contundente como un antropólogo comprometido con los sujetos de estudio y que será la directriz principal de su trayectoria como antropólogo social. Su trabajo en el proyecto de la Cuenca del Papaloapan de los años cincuenta, con otros colegas estudiantes y pasantes, fue de los primeros estudios etnográficos comunitarios de las poblaciones mazatecas y chinantecas que serían afectadas por la construcción de la Presa Miguel Alemán. En éste deja ver su análisis crítico y los señalamientos de la falta de criterios que protejan los derechos humanos individuales y sociales de los pueblos mazatecos afectados, que serían cuidadosamente realizados y orientados críticamente por Julio de la Fuente y Gonzalo Aguirre Beltrán.

Esta obra, aunada a la elaborada diez años después, en 1963, sobre el "Desarrollo agrícola y las clases sociales en África”, publicada por El Colegio de México, junto a su trabajo de sociología del campo en Brasil, nuevamente refleja la importancia de la etnografía del trabajo de campo para la investigación social en América Latina. Podríamos revisar cada uno de los artículos de la excelente y gran contribución académica de Rodolfo para distinguir la articulación profunda entre antropología y sociología, donde en el caso mexicano la primera se convierte en el germen para el surgimiento de la segunda. Como muestra está el auditorio de la Facultad de Ciencias Políticas y Sociales que lleva el nombre de Lucio Mendieta y Núñez, fundador del Instituto de Investigaciones Sociales y de la Escuela de Sociología de la UNAM, quien trabajó directamente con Manuel Gamio en el estudio regional del Valle de Teotihuacán en 1915, que culmina en 1922 con su publicación por la Dirección de Agricultura.

\section{LA ANTROPOLOGÍA ANALÍTICA Y APLICADA EN LA OBRA DE RODOLFO STAVENHAGEN '}

Así como la antropología nutre a la sociología y ambas se retroalimentan, Rodolfo Stavenhagen tiene un gran peso en la construcción de las ciencias sociales en México. Nuestro homenajeado no separa el trabajo antropológico del sociológico, porque a lo largo de su vida va construyendo un puente para el diálogo, para lograr una interacción y una fusión entre ambas disciplinas de las ciencias sociales. Lo mismo escribe un artículo en 1967 sobre los problemas de las ciencias sociales en América Latina, en el que ya tiene una propuesta analítica relacional entre antropología social y sociología y que, a mi entender, consolidará en su libro publicado en 1969: Las clases sociales en las sociedades agrarias (1978a), que será un referente permanente tanto en el campo de la antropología como en el de la sociología. Este libro es construido a partir de una objetividad académica de alta preeminencia, dado que es la época de la discusión controversial entre el marxismo y las

\footnotetext{
${ }^{1}$ Rodolfo Stavenhagen fue miembro del Consejo Directivo de la Society for Applied Anthropology y contó con amplio reconocimiento en dicha sociedad.
} 
corrientes sociológicas y antropológicas que, para el caso que señalamos, se manifiestan con un equilibrio analítico de gran relevancia sin generar polarizaciones que violenten los derechos humanos por los cambios sociales que deben suceder en América Latina. Esta posición bastante compleja en las ciencias sociales no deja duda del posicionamiento de Rodolfo en cuanto a su planteamiento progresista y de gran avanzada, sin polarizar los análisis académicos, lo que le conferirá un gran reconocimiento a nivel internacional y nacional por su destacado equilibrio académico, que muchos colegas reiteramos y que hoy se considera parte de la aportación científica en el campo de las ciencias sociales de un mexicano destacado como Rodolfo Stavenhagen.

Anoté que en el Boletín del INAH en 1973 escribe un breve artículo que denomina "Una ciencia social, crítica, radical y comprometida" (Stavenhagen, 1973b), en el cual revela su posicionamiento académico y político, que después desarrolla en el discurso que ofrece al recibir el Premio Elías Sourasky de de 1978 surge una nueva reflexión en los discursos analíticos de Rodolfo, al advertir la necesidad de enfocar la atención de las ciencias sociales hacia otro desarrollo con enfoques y estrategias. Publica estos pensamientos en 1978 en la editorial Siglo XXI $\mathrm{y}$, desde mi punto de vista, los problemas étnicos y los postulados campesinistas empiezan a tener una gran relación en su análisis. En 1980, en el libro Problemasétnicosy campesinos: ensayos (Stavenhagen, 1980), publicado por el Instituto Nacional Indigenista (INI), ya se define entre la postura campesinistamarxista y la que este grupo denominará "etnicista", en referencia a la posición de la defensa de los pueblos originarios de México y de América Latina, a los cuales Rodolfo alude con un equilibrio analítico muy sólido. También manifiesta su orientación hacia "La cultura popular y la creación intelectual en México" (Stavenhagen, 1982) como director general de Culturas Populares de la Secretaría de Educación Pública (SEP), al dar gran relevancia a esta actividad en la sociedad mexicana y en todos los estados de la república.

\section{LA ANTROPOLOGÍA CRÍTICA Y LOS DERECHOS HUMANOS UNIVERSALES Y DE LOS PUEBLOS INDÍGENAS DE MÉXICO, DEL CONTINENTE Y DEL MUNDO}

Es importante señalar como una aportación a la antropología el trabajo de Rodolfo sobre la cuestión étnica y las ciencias sociales que publicó en el Journal of Cultures and Ideas en la ciudad de Lagos, Nigeria, dentro del Círculo de Estudios Sociales en África, en el que resalta la orientación de la antropología que tiene un peso significativo. A partir de ese año la visión etnológica predominará en los estudios que se proyectan en todos sus trabajos, hasta la creación del concepto de "etnodesarrollo". Este concepto adquirirá relevancia en México y modificará de manera sustantiva la política indigenista integracionista, a partir de 1985. Dicho planteamiento fue promovido por Rodolfo a través de la unesco, en una reunión convocada específicamente en el Consejo Latinoamericano de Ciencias Sociales, en Costa Rica, en 1982. Ayudó a reformular la política indigenista en México en 1983 -que me tocó impulsar como director del INI $-{ }^{2}$ y que llevó al gobierno mexicano a tratar de frenar dicha posición, que con el tiempo generó el levantamiento armado zapatista de 1994. De este conflicto, Rodolfo presidió la Comisión de Seguimiento y Verificación de los Acuerdos de San Andrés Larráinzar, desafortunadamente no concluidos. El levantamiento armado, en el fondo, refleja los conflictos étnico y de los derechos humanos, no sólo en México, sino en el mundo, sobre los cuales Rodolfo publicó "Conflictos étnicos y derechos humanos. Sus interrelaciones" (Stavenhagen, 1987).

Considero que desde ese momento la orientación de Rodolfo destacará en su lucha por lograr que la ONU estableciera la discusión en el seno de la

\footnotetext{
2 Por tratar de instrumentar en México un cambio sustantivo de la política indigenista del integracionismo al etnodesarrollo se generó una gran controversia al interior del gobierno que me llevó a la cárcel en octubre de 1983, y gracias al apoyo de Rodolfo Stavenhagen pude obtener mi libertad.
} 
Asamblea General para que la Declaración Universal de los Derechos de los Pueblos Indígenas fuera aceptada plenamente, hecho que se concretó hasta 2007 , en que fue aprobada y que hoy rige las relaciones entre los gobiernos de todos los países que la han ratificado y los pueblos indígenas del mundo. Rodolfo publica el relevante trabajo Conflictos étnicos y Estado nacional en el año 2000 (Stavenhagen, 2000b), con una visión universal, que revela cómo en varias regiones del mundo hay conflictos étnicos y cómo se resuelven en algunos países y en otros permanecen latentes a pesar de los años transcurridos. Es una obra colectiva entre distinguidos investigadores que analizaron la situación de Senegal, Guatemala, Líbano, Burundi —en el cuerno de África-, India, Nigeria, Guyana, las Islas Fiji, Malasia, Pakistán, Unión Soviética, Yugoslavia y los Estados Unidos, lo que da testimonio de la visión universalista de Rodolfo y de su enorme capacidad de coordinar a investigadores de todos esos países para publicar esta obra de trascendencia internacional.

En este proceso de aprobación pública en distintos países de América Latina y del mundo sobre las perspectivas de los derechos indígenas en el sistema internacional, Rodolfo Stavenhagen tiene un papel de alta relevancia como primer relator especial sobre la situación de los derechos humanos y las libertades fundamentales de los pueblos indígenas del Consejo de Derechos Humanos de la onU. Si revisamos con detenimiento los informes publicados por la oficina de la unesco en México (Stavenhagen, 2008), que incluyen los reportes de 2002 al 2007, cada uno contiene una amplia descripción etnográfica sobre la situación que aqueja a los pueblos indígenas del mundo, que finalmente condujo a la votación de 143 Estados miembros de Naciones Unidas del 13 de septiembre de 2007 a favor de adoptar la Declaración Universal de los Derechos de los Pueblos Indígenas, en la que sólo cuatro países votaron en contra - Australia, Canadá, Estados Unidos y Nueva Zelandia-. Dicha declaración recoge las aspiraciones de los pueblos originarios del mundo y establece las normas que deben seguirse desde esa fecha en adelante para la protección de los derechos humanos de las personas y las comunidades indígenas, misma que contiene 46 artículos que cubren todos los derechos civiles, políticos, económicos, sociales y culturales que forman parte de todos los instrumentos internacionales de derechos humanos. Desde mi punto de vista, éste es uno de los logros más importantes de este inicio del siglo XxI, el cual sienta los principios fundamentales para la eliminación del colonialismo, la explotación, la discriminación racial y cultural y, sobre todo, busca la igualdad de derechos para todos los seres humanos y para todos los pueblos originarios de cada una de las entidades nacionales.

\section{Revisión universal de los derechos de los pueblos indígenas y las minorías étnicas desde el papel de relator}

La lucha del doctor Stavenhagen durante siete años queda registrada como una de las aportaciones más importantes en que las ciencias sociales reconocen y afirman a los pueblos originarios e indígenas del mundo. En cada uno de los informes redactados por el relator especial se proyectan la gran experiencia y el rigor académico con recomendaciones puntuales basadas en visitas de trabajo de campo directas en el terreno donde viven las gentes y los pueblos indígenas, como un elemento básico para producir informes detallados que pueda constatar el relator. Siguiendo los métodos de la antropología, Rodolfo aplicó su alta responsabilidad como relator para visitar distintas regiones del mundo, donde mantuvo una comunicación fluida con las organizaciones indígenas y recibió comunicaciones de particulares e instituciones en relación con sus derechos humanos y sus libertades fundamentales. Estas visitas produjeron documentos cuantitativos complementados con una base de datos que se construyó durante siete años, en la que sobresalen las comunicaciones recibidas sobre las presuntas violaciones de los derechos humanos de 
los indígenas. Con estos informes, la antropología como ciencia social especializada en la diversidad cultural y humana marca el principio de cambios fundamentales en la relación de los pueblos indígenas con las Naciones Unidas y con sus propios países como instrumentos jurídicos y de derechos humanos para cambiar las graves condiciones en que se encuentran. Baste leer estos informes para reconocer la gran aportación humanitaria y científica de Rodolfo Stavenhagen, un gran humanista mexicano que merece este gran reconocimiento de la UNAM y en especial de las instituciones de ciencias sociales.

\section{BIBLIOGRAFÍA}

Bonfil Batalla, Guillermo, 1982, América Latina: etnodesarrollo y etnocidio, Facultad Latinoamericana de Ciencias Sociales, San José.

Chazarreta, Adriana, 2010, "La propuesta analítica de Rodolfo Stavenhagen", en Prácticas de Oficio. Investigación y Reflexión en Ciencias Sociales, núm. 6.

Gómez, Magdalena, 2011, "Pueblos indígenas y derechos humanos", en La Jornada, 15 de marzo, en línea: <http:// www.jornada.unam.mx/2011/03/15/politica/022a1pol>.

Stavenhagen, Rodolfo, 1953, "Aspectos de la antropología social aplicada en la Cuenca del Papaloapan", en Tlatoani, núm. 7, Escuela Nacional de Antropología e Historia, México.

_ 1963, "Desarrollo agrícola y las clases sociales en África”, en Foro Internacional, vol. IV, núm. 12, El Colegio de México, México.

—, 1964 , "La sociología del campo en Brasil”, en Foro Internacional, vol. IV, núm. 4, El Colegio de México, México.

- 1968, Neolatifundismo y explotación, de Emiliano Zapata a Anderson Clayton and Co., Nuestro Tiempo, México.

- 1970, Agrarian Problems and Peasant Movements in Latin America, Doubleday and Company, Inc., Nueva York.

— 1973a, "El compromiso de las ciencias sociales en México”, en Diálogos, núms. 49-60, El Colegio de México, México.

_ 1973 b, "Una ciencia social crítica, radical y comprometida", en Boletín del INAH, pp. 33-34.
—, 1974 , Sociología y subdesarrollo, Nuestro Tiempo, México.

- 1978a, Las clases sociales en las sociedades agrarias, Siglo XXI, México.

— 1978b, Testimonios, Universidad Nacional Autónoma de México, México.

— 1980 , Problemas étnicos y campesinos: ensayos, Instituto Nacional Indigenista, Secretaría de Desarrollo Social, México.

— 1982, "La cultura popular y la creación intelectual", en Adolfo Colombres (comp.), La cultura popular, Premiá Editores, México, pp. 21-39.

- 1983, "The Ethnic Question and the Social Sciences", en Journal of Cultures and Ideas, vol. I, núm. 1, Alada Study Circle of Nigeria, Lagos, pp. 115-132.

—, 1984, Imagen y obra escogida, Universidad Nacional Autónoma de México, México.

_ 1987, "Ethnic Conflict and Human Rights. Their Interrelationship", en Bulletin of Peace Proposals, vol. 18, núm. 4, Peace Research Institute-Oslo, Norwegian University Press, pp. 507-514.

- 1988, Política cultural para un país multiétnico, Secretaría de Educación Pública, Ediciones El Caballito, México.

- 1990, Entre la ley y la costumbre, el derecho consuetudinario indígena en América Latina, Instituto Indigenista Interamericano, Instituto Interamericano de Derechos Humanos, México.

— $2000 \mathrm{a}$, Derechos humanos de los pueblos indígenas, Comisión Nacional de Derechos Humanos, México.

— 2000b, Conflictos étnicos y Estado nacional, Siglo XXI, México.

- - 2008, Los pueblos indígenas y sus derechos. Informes temáticos del relator especial sobre la situación de los derechos humanos y las libertades fundamentales de los pueblos indígenas del Consejo de Derechos Humanos de la Organización de las Naciones Unidas, Organización de las Naciones Unidas para la Educación, la Ciencia y la Cultura, México.

-, 2011, "México en el bicentenario: la ciudadanía indígena a debate", en Revista Andaluza de Antropología, núm. 1, pp. 86-98.

, 2012, "Multiculturalismo y buen vivir. Desafíos ante la crisis actual", ponencia, Reunión de la Academia Mexicana de Ciencias, Academia Mexicana de Ciencias, México.

Zapata, Francisco, 1995, "Las siete tesis: treinta años después”, en Estudios Sociológicos, núm. 13, pp. 181-187. 